\title{
Laser Scanner-Stage Synchronization Method for High-Speed And Wide-Area Fabrication
}

\author{
Kyunghan $\mathrm{Kim}^{{ }^{* 1}}$, Kwangho Yoon ${ }^{{ }_{1}}$ Jeong Suh $^{{ }^{* 1}}$, and Jaehoon Lee ${ }^{{ }^{* 1}}$ \\ ${ }^{*}$ Dept. of High Density Energy Beam Processing \& System, Korea Institute of Machinery \& \\ Materials, 104 Sinseongno, Yeseong- gu, Dae-jeon, 305-343, Korea \\ E-mail: jaholee@kimm.re.kr
}

\begin{abstract}
This paper presents an on-the-fly method to synchronize a laser galvanometer scanner and linear stage for fast and wide-area fabrication. The location and velocity information of the linear stage is transferred to the galvanometer scanner control board by an encoder signal. The scanner control board calculates the amount of laser beam movement by subtracting from original CAD data to linear stage movement. The on-the-fly method is different from the existing step and scanning method in that it ensures continuous stage movement and real-time signal transfer between the linear stage and galvanometer scanner.
\end{abstract}

Keywords: Laser, Galvanometer scanner, Linear stage, On-the-fly, Synchronization DOI:10.2961/jlmn.2012.02.0018

\section{Introduction}

Short-pulsed laser technology allows for material fabrication processes using ultra-precision lasers, such as viahole drilling [1], FPCB (Flexible PCB) cutting [2], and surface texturing [3]. This trend of laser fabrication requires a high-speed, wide-area, and dry process due to concerns over environmental problems. The galvanometer scanner is being widely used as a marking tool [4]. Recently, it has been applied to many laser material fabrication fields [5]. However, the working field size of this scanner is limited by the focal length of the f-theta objective lens, which is measured from the scanner head to the sample. When the focal length is increased, the working field size of the scanner becomes wider, but the resolution of the scanning field decreases, which can be a drawback for precise fabrication. A hybrid method that involves the use of both a linear stage and a scanner is being considered for wide-area fabrication. The initial approach that has been adopted is known as the step and scanning method. The stage repeatedly moves and stops in steps, and the scanner operates when the stage stops. Therefore, this method causes discontinuous quality and increases the fabrication time, because stage motion stops when the scanner is operating. To overcome this problem, a hybrid method that involves the use of both a linear stage and a scanner has recently been developed. Manufacturers of the scanner-stage synchronization processing equipment include ESI, LPKF, and AEROTECH. Electro Scientific Industries (ESI) applied on-the-fly equipment to micro via-hole drilling and FPCB cutting. The ESI UV laser drilling system HDI 5330 is capable of on-the-fly processing with a $533 \mathrm{~mm} \times 635 \mathrm{~mm}$ working area; this system can guarantee $20 \mu \mathrm{m}$ accuracy with a $500 \mathrm{~mm} / \mathrm{s}$ fabrication speed. LPKF of Germany recently released the MicroLine 6000 model for cover layer cutting of PCB or FPCB. The company provides software known as LPKFCAM, which profiles the stage path and scanner working path and reduces the fabrication time by $20 \%$. In recent years, AEROTECH of the United States has developed similar on-the-fly equipment with the preprocessing path optimization software Automation 3200 Some examples of on-the-fly equipment are shown in Fig. 1 .

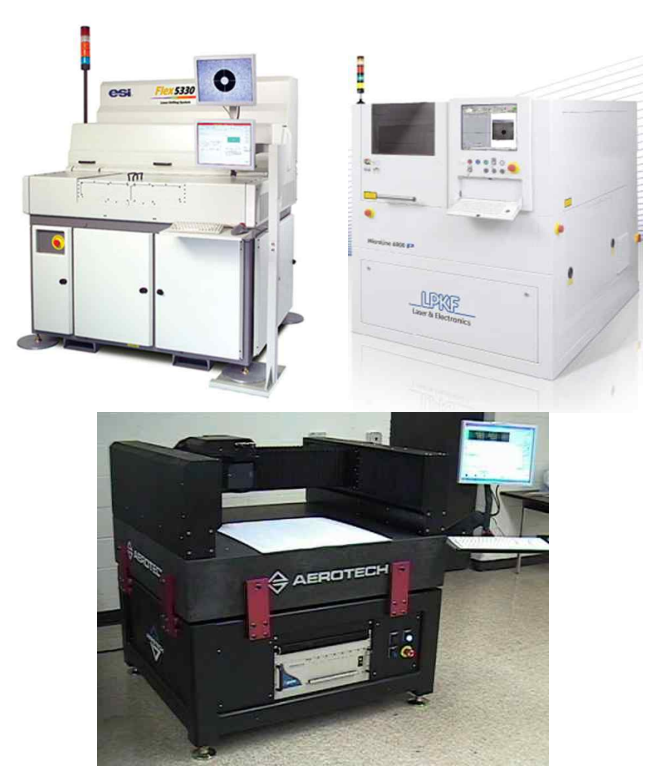

Fig. 1 Photos of on-the-fly equipment of several companies: ESI, LPKF, and AEROTECH.

Some commercial scanner control boards provide onthe-fly applications with options, such as the RTC 5 board of SCANLAB and the marking on-the-fly (MOTF) add-on board of RAYLASE. However, the main application of the MOTF board is limited to marking of the serial number, time, and so on, of large-area electronic parts in the conveyor feed stage. In this study, we mainly focused on ap- 
plication to FPCP cover layer cutting; the work can be summarized as follows:

(1) Stage path and velocity profiling software development for preprocessing

(2) On-the-fly control board development for laser fabrication purposes

(3) On-the-fly system buildup with a linear stage

(4) On-the-fly system validation by experiments

\section{On-the-fly system configuration}

\subsection{Stage path/velocity profiling}

The on-the-fly control system is composed of stage path/velocity profiling software, a scanner control board, and a stage control board. First, the tool path for laser fabrication needs to be determined by the stage path profiling algorithm. In the $\mathrm{CAD}$ data, the stage allocates a rough path because its velocity and acceleration are small compared to the scanner's velocity and acceleration. For the detail region, which is commonly called the high-frequency region in CAD data, the scanner works because the galvanometer mirror system in the scanner enables delivery of the laser beam with almost zero inertia. As shown in Fig. 2, the stage path is smoothed into a parabolic shape to minimize the stage acceleration and deceleration when fabricating a $90^{\circ}$ corner line. The radius of curvature of the stage path is determined by the stage acceleration time (Ta). If the value of the stage acceleration time is large, the stage path becomes smoother. An example of stage path profiling is shown in Fig. 3. To fabricate targeting CAD data, the stage path can be determined by the variations in $\mathrm{Ta}$. If $\mathrm{Ta}$ changes from $60 \mathrm{~ms}$ to $80 \mathrm{~ms}$, the stage path becomes smooth, and the stage movement decreases.

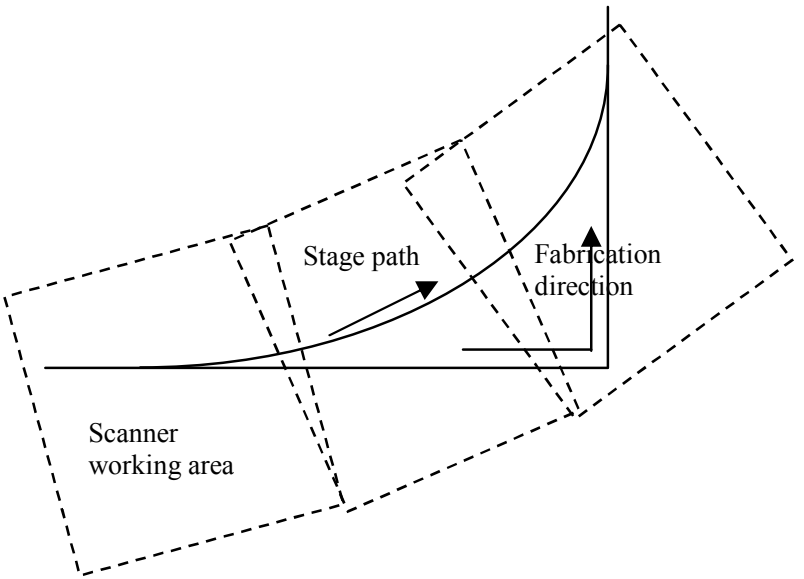

Fig. 2 Example of stage path profiling

Once the stage path is determined, the scanner working path can be calculated from the vector relationship, as shown in Fig. 4. The fabrication movement vector $\left(\vec{P}_{f a, i}\right)$ is the summation of the stage movement vector $\left(\vec{P}_{s t, i}\right)$ and scanner movement vector $\left(\vec{P}_{s c, i}\right)$ for a certain period and is defined by Eq. (1) as follows:

$$
\vec{P}_{s t, i}+\vec{P}_{s c, i}=\vec{P}_{f a, i}
$$

To synchronize the scanner stage, the duration of each movement vector should be

$$
\frac{\left|\vec{P}_{s t, i}\right|}{v_{s t, i}}=\frac{\left|\vec{P}_{s c, i}\right|}{v_{s c, i}}=\frac{\left|\vec{P}_{f a, i}\right|}{v_{f a, i}}
$$

where the stage velocity $\left(v_{s t, i}\right)$ and scanner velocity $\left(v_{s c, i}\right)$ can be calculated by Eq. (3) as follows:

$$
\begin{aligned}
& v_{s t, i}=\frac{\left|\vec{P}_{s t, i}\right|}{\left|\vec{P}_{f a, i}\right|} \times v_{f a, i} \\
& v_{s c, i}=\frac{\left|\vec{P}_{s c, i}\right|}{\left|\vec{P}_{f a, i}\right|} \times v_{f a, i}
\end{aligned}
$$
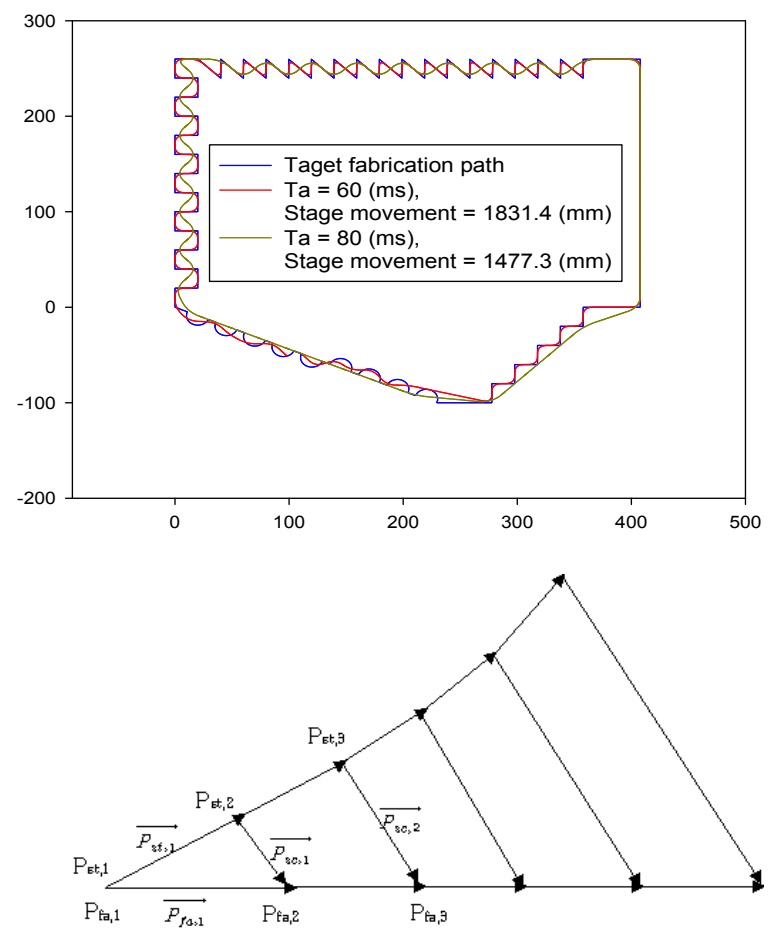

Fig. 4 Vector decomposition of each component path

\subsection{On-the-fly control board development}

The stage path and velocity are determined by the PC level using position profiling and preprocessing. The information obtained from the stage position and velocity is then transferred to the stage control board. The stage motion and speed are controlled by the stage driver command. The information obtained from the stage location and velocity is also transferred to the scanner control board with an encoder signal, as shown in Fig. 5. In the scanner control board, the scanner movement and velocity are calculated using the CAD data and the stage path and velocity. The stage movements may not follow the command, and the stage tracking error is also added or subtracted in the scanner control board. 


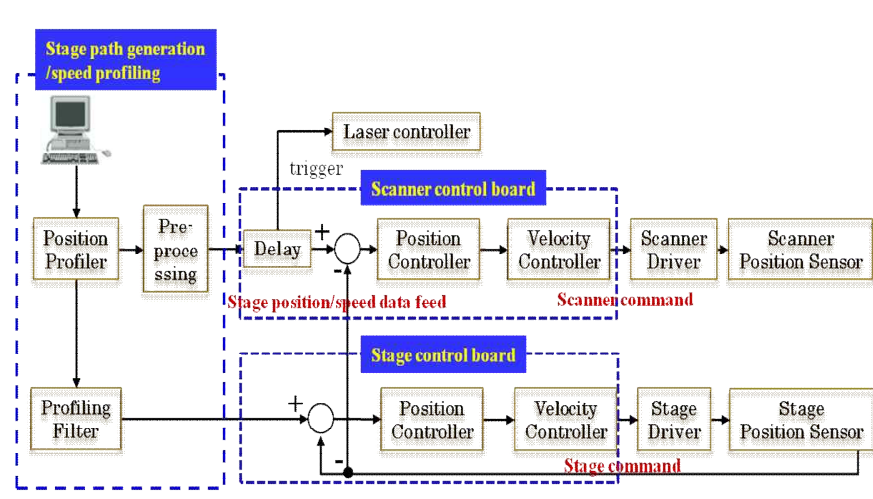

Fig. 5 On-the-fly control system layout

\subsection{Comparison of on-the-fly method with step and scanning method}

Initially, a one-axis stage-two-axis scanner on-the-fly system was developed and tested, as shown in Fig. 6. An IPG fiber laser with a $30 \mathrm{~ns}$ pulse width, $12 \mathrm{~W}$ power, and $1064 \mathrm{~nm}$ wavelength was used for the on-the-fly marking experiment. A CTI scanner head with an aperture of $10 \mathrm{~mm}$ and focal length of $100 \mathrm{~mm}$ was used. The stage of DASAROBOT has a 400-mm stroke with a maximum speed of $500 \mathrm{~mm} / \mathrm{s}$ and a precision of approximately $5 \mu \mathrm{m}$.

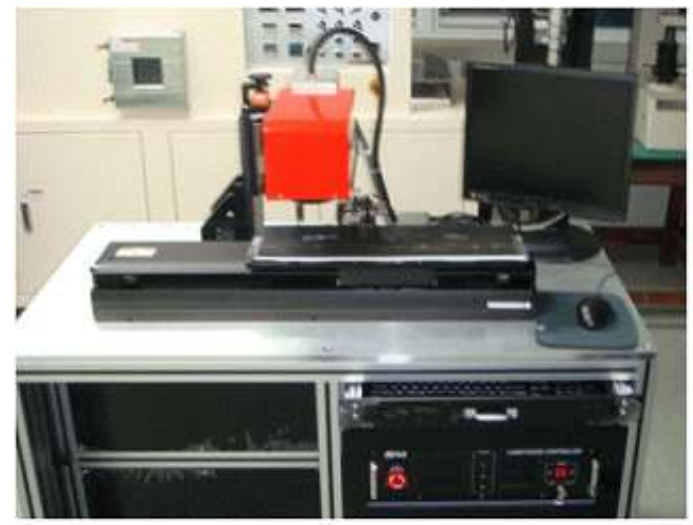

Fig. 6 Photo of one-axis stage-two-axis scanner on-the-fly system

The marking precisions of the step and scanning method and on-the-fly method were compared at a low pulse repetition rate of $1 \mathrm{kHz}$. The marking speed was $300 \mathrm{~mm} / \mathrm{s}$, and the scanner working area was $50 \mathrm{~mm} \times 50 \mathrm{~mm}$. In the step and scanning method, the stage stops when the scanner is operating. After the scanner operates within the working area, the stage shifts $50 \mathrm{~mm}$ to the right to begin with the next process. The scanning and step movement process is repeated to mark the entire area. However, in the on-the-fly system, the stage and scanner move and work together. Various marking directions from $0^{\circ}$ to $90^{\circ}$ were tested, as shown in Fig. 7. Three areas were selected to compare the marking qualities between the two methods.

Figure 8 compares the two methods at the marked spots. In the scanner working area of both methods, the distances between the marking spots were almost constant in all directions. In the step and scanning method, irregular spots were observed at the boundary of the scanner working area. This is because the exact marking positions could not be controlled owing to the irregular start and stop movements of the stage. However, the on-the-fly method showed an almost constant marking distance between spots in the boundary region and working area. This is a critical advantage of the on-the-fly system over the step and scanning method.

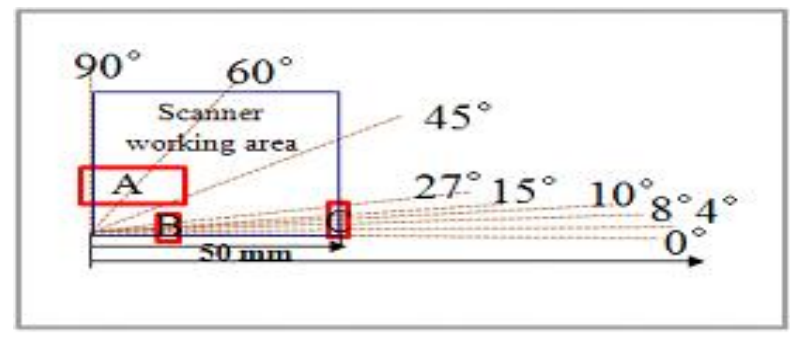

Fig. 7 Experimental scenario of laser marking test for comparison between step and scanning and on-the-fly methods
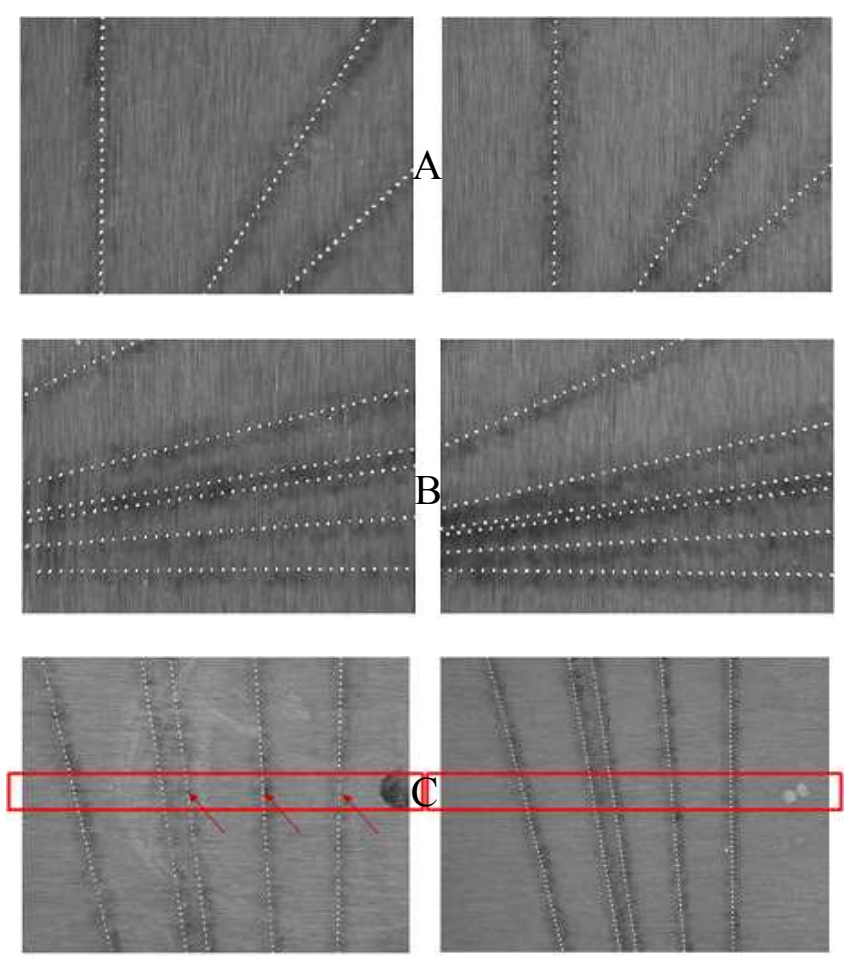

Fig.8 Results of laser marking pattern of two methods: step and scanning method (left) and on-the-fly method (right)

\subsection{Accuracy measurement of on-the-fly system}

Recently, we expanded the one-axis linear stage in the on-the-fly system to a two-axis linear stage. The scanner control board can input encoder signals to both the $\mathrm{x}$ and $\mathrm{y}$ axes of the stage motion board. The hardware was built up with a two-axis gantry linear stage having a $\mathrm{Z}$-axis linear stage, as shown in Fig. 9. 


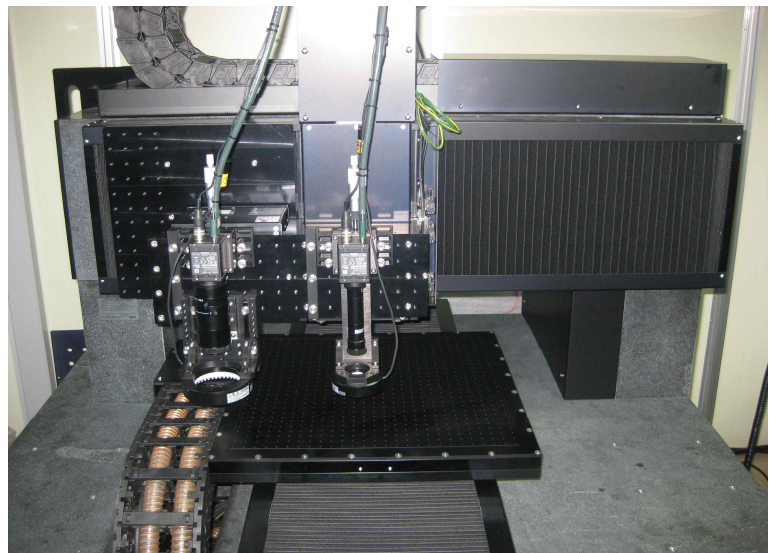

Fig. 9 Photo of two-axis stage and two-axis scanner onthe-fly system.

Each axis of the linear stage was tested and measured for accuracy by a laser interferometer made by RENISHAW. The repeatability was measured to be $0.4 \mu \mathrm{m}$ for the $\mathrm{x}$ axis and $0.29 \mu \mathrm{m}$ for the y axis over the entire work area of 500 $\mathrm{mm} \times 500 \mathrm{~mm}$. The accuracy of the linear stage was measured to be $3.03 \mu \mathrm{m}$ along the $\mathrm{x}$ axis and $1.29 \mu \mathrm{m}$ along the y axis.

Initially, the accuracy of the system was measured without synchronization of the galvanometer and two-axis linear stage. To measure the accuracy of the scanner system itself, a square shape with a length of $40 \mathrm{~mm}$ on one side was marked when the linear stage stopped. The error of the scanner system is summarized in Table 1. The maximum error of the side length was less than $8 \mu \mathrm{m}$, and the average error of the four side lengths was $2.5 \mu \mathrm{m}$.

Table 1. Error of galvanometer scanner when marking a square with a side length of $40 \mathrm{~mm}$.

\begin{tabular}{|c|c|c|c|c|c|}
\hline & \multicolumn{2}{|c|}{ Position $(\mathrm{mm})$} & \multirow{2}{*}{\multicolumn{2}{|c|}{$\begin{array}{l}\text { Side length } \\
(\mathrm{mm})\end{array}$}} & \multirow{2}{*}{$\begin{array}{l}\text { Error } \\
(\mu \mathrm{m})\end{array}$} \\
\hline & $\mathrm{x}$ & $\mathrm{y}$ & & & \\
\hline 1 & 0 & 0 & S1 & 40.000 & 0 \\
\hline 2 & 0 & 40 & S2 & 39.997 & -3 \\
\hline 3 & 39.997 & 40.005 & S3 & 40.008 & 8 \\
\hline 4 & 40.005 & -0.003 & S4 & 40.005 & 5 \\
\hline & & Iverage & & 40.0025 & 2.5 \\
\hline
\end{tabular}

The galvanometer scanner and linear stage were then synchronized, and the wide area was marked by a square shape with a side length of $350 \mathrm{~mm}$. The test results are summarized in Table 2. The maximum deviation of the side length was $8 \mu \mathrm{m}$, which is similar to the summation of the linear stage and scanner errors. However, the error for the average side length of the large rectangle was still less than $5 \mu \mathrm{m}$. The maximum angular error occurred between M3 and M4 and was measured to be $0.0025^{\circ}$, which is a quite trivial value. For this marking test with the on-the-fly system, the error included both of the stage and scanner errors. Again, information of the position and velocity of the linear stage was transferred to the scanner control board through the encoder signal. At the scanner control board, the stage position was corrected by a comparison with reference CAD data. Even though the corrected information was commanded by the scanner control board, the galvanometer system can have error sources: for example, optical distor- tions and mechanical error. The test results for the accuracy of the scanner system and on-the-fly system are shown in the Fig. 10.

Table 2. Error of the on-the-fly system when marking a square with a side length of $350 \mathrm{~mm}$.

\begin{tabular}{|c|c|c|c|c|c|}
\hline \multirow[b]{3}{*}{1} & \multicolumn{2}{|c|}{ Position (mm) } & \multirow{2}{*}{\multicolumn{2}{|c|}{$\begin{array}{l}\text { Side length } \\
(\mathrm{mm})\end{array}$}} & \multirow{2}{*}{$\begin{array}{l}\text { Error } \\
(\mu \mathrm{m})\end{array}$} \\
\hline & $\mathrm{x}$ & $\mathrm{y}$ & & & \\
\hline & 0 & 0 & M1 & 350.000 & 0 \\
\hline 2 & -0.005 & 350 & M2 & 350.008 & 8 \\
\hline 3 & 350.003 & 349.993 & M3 & 350.003 & 3 \\
\hline 4 & 350.007 & -0.010 & M4 & 350.007 & 7 \\
\hline & & Iverage & & 350.0045 & 4.5 \\
\hline
\end{tabular}

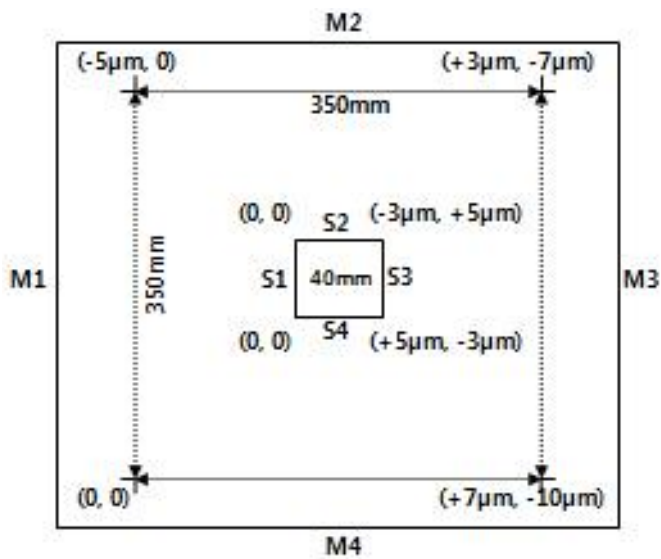

Fig. 10 Results for the position accuracy of the scanner and the on-the-fly system: the small square in the center represents the scanner system error, and the outside square represents the on-the-fly system position error.

The on-the-fly system accuracy was then tested by synchronization of the two systems. Three equilateral triangles with side lengths of 100,200 , and $300 \mathrm{~mm}$ were marked at a $350 \mathrm{~mm} / \mathrm{s}$ marking speed, and the three sides and angles of each triangle were measured. The error of the side length was measured to be about $5 \mu \mathrm{m}$ and was quite similar for all three triangles.

However, the side lengths of L1 and L2 show deviated results from nominal value all three cases. One side length may have been overestimated, and the other side may have underestimated the measuring interception point between L1 and L3. Another possibility may be that the stage control by the UMAC company was not finely tuned enough to make the cross line L2. The largest angular error was at a1. All angular errors were measured and found to be less than $0.05^{\circ}$ in the three triangle cases. The test results are summarized in Table 3.

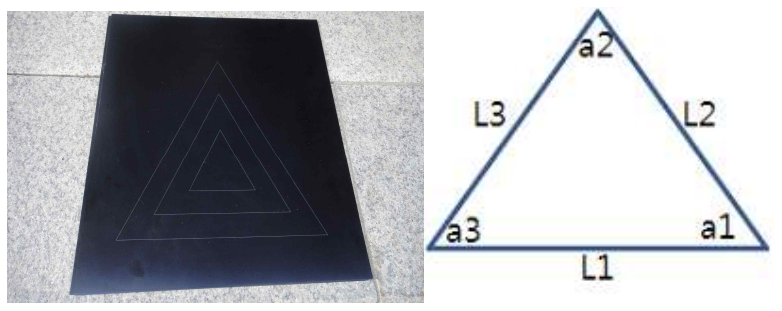

Fig. 11 Marking results of three triangles (left) and measurement points (Right) 
Table 3. Error of on-the-fly system when marking three different triangles.

\begin{tabular}{|c|c|c|c|}
\hline & $\begin{array}{c}100-\mathrm{mm} \\
\text { Triangle }\end{array}$ & $\begin{array}{c}\text { 200-mm } \\
\text { Triangle }\end{array}$ & $\begin{array}{c}\text { 300-mm } \\
\text { Triangle }\end{array}$ \\
\hline $\mathrm{L} 1(\mathrm{~mm})$ & 100.06704 & 200.1119 & 300.16525 \\
\hline $\mathrm{L} 2(\mathrm{~mm})$ & 99.94538 & 199.89254 & 299.84076 \\
\hline L3 $(\mathrm{mm})$ & 100.00391 & 200.01105 & 300.01027 \\
\hline $\begin{array}{c}\text { Average } \\
(\mathrm{mm})\end{array}$ & 100.00544 & 200.00516 & 300.00543 \\
\hline Error $(\mu \mathrm{m})$ & 5.44 & 5.16 & 5.42 \\
\hline $\mathrm{a} 1\left(^{\circ}\right)$ & 59.9972 & 59.9965 & 59.9944 \\
\hline $\mathrm{a} 2\left(^{\circ}\right)$ & 60.0624 & 60.0593 & 60.0600 \\
\hline $\mathrm{a} 3\left(^{\circ}\right)$ & 60.0639 & 60.0564 & 60.0584 \\
\hline Average $\left(^{\circ}\right)$ & 60.0412 & 60.0374 & 60.0376 \\
\hline Error $\left(^{\circ}\right)$ & 0.041 & 0.037 & 0.038 \\
\hline
\end{tabular}

\section{Conclusion}

An on-the-fly method was developed to synchronize the galvanometer scanner and linear stage. The stage path and velocity were determined at the PC level by position profiling preprocessing. The stage path was determined from the stage acceleration value. When Ta was increased, the stage path became smoother and shorter. The information obtained from the stage path and velocity was transferred to both the stage control driver and the scanner. On the scanner control board, the amount of scanner movement was calculated from the difference between the CAD data and the stage movement information. In the one-axis stage-two-axis scanner on-the-fly system, the marking distances between certain spots were measured. Almost constant distances were observed at both the scanner working area and the boundary region. However, in the step and scanning method, irregular marking points were observed at the boundary of the scanning area, which may affect the quality of fabrication.

The on-the-fly system was expanded to the two-axis linear stage and two-axis scanner system. First, the twoaxis linear stage was evaluated for its repeatability and accuracy through the use of a laser interferometer. The accuracy of the linear stage was measured to be about $3 \mu \mathrm{m}$. The galvanometer scanner error was about $2.5 \mu \mathrm{m}$. The maximum error of the on-the-fly system was then measured to be less than $10 \mu \mathrm{m}$ when marking a wide-area square shape. The precision test was expanded to mark triangles with different sizes. The length error of the side was measured to be about $5 \mu \mathrm{m}$, and the angular error was measured to be less than $0.05^{\circ}$; these results were almost independent of the triangle size. The precision of the developed on-thefly system was well-validated by various marking tests. The maximum error of the developed on-the-fly system almost corresponded to the current state-of-art on-the-fly system, which has an accuracy of $20 \mu \mathrm{m}$ with a $500 \mathrm{~mm} / \mathrm{s}$ fabrication speed. In the future, we will develop a highspeed on-the-fly system with high accuracy.

\section{Acknowledgments and Appendixes}

This work was supported by the Korean Ministry of Knowledge Economy within the project "Precision/High-
Speed Laser Cutting System Core Technology Development" (Industrial Source Technology Development, Project Number: 10033829) and "Development of next generation multi-functional machining system for eco/bio components" (Industrial Source Technology Development, Project Number: 10039982)

\section{References}

[1] Zhang C., Salama I. A., Quick N. R., and Kar A: J. Physics D: Appl. Phys, 39, (2006) pp. 3910-3918.

[2] Shin D., Lee J., Sohn H., Noh J., and Paik B., JLMN, Vol. 5, No. 1, (2010) pp. 48-52.

[3] Khan Malek C. G., Vol. 385,(2006), pp.1362-1369

[4] Kang H. J., Kim H. J., Kim J. S., Choi. W. Y., Chu. W. S., and Ahn. S. H., Robotics and Computer-Integrated Manufacturing (in press).

[5] Avinash K. D. and Vinod Y., J. Mater. Process. Technol., Vol. 195, (2008), pp. 15-26.

(Received: July 08, 2011, Accepted: April 11, 2012) 